\title{
Catalytic Properties of Vanadium Diselenide: A Comprehensive Study on Its Electrocatalytic Performance in Alkaline, Neutral, and Acidic Media
}

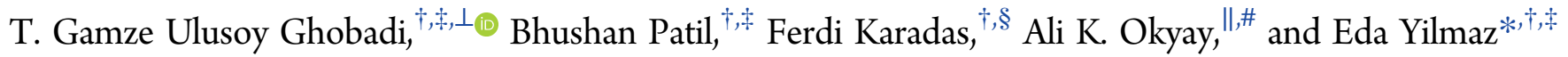 \\ ${ }^{\dagger}$ UNAM-National Nanotechnology Research Center, ${ }^{\ddagger}$ Institute of Materials Science and Nanotechnology, ${ }^{\S}$ Department of \\ Chemistry, and "Department of Electrical and Electronics Engineering, Bilkent University, 06800 Ankara, Turkey \\ ${ }^{\perp}$ Department of Energy Engineering, Faculty of Engineering, Ankara University, 06830 Ankara, Turkey
}

Supporting Information

\begin{abstract}
Here, we report the synthesis of vanadium diselenide $\left(\mathrm{VSe}_{2}\right)$ three-dimensional nanoparticles (NPs) and two-dimensional (2D) nanosheets (NSs) utilizing nanosecond pulsed laser ablation technique followed by liquid-phase exfoliation. Furthermore, a systematic study has been conducted on the effect of NP and NS morphologies of $\mathrm{VSe}_{2}$ in their catalytic activities toward oxygen reduction reaction (ORR), oxygen evolution reaction (OER) and
\end{abstract} hydrogen evolution reaction (HER) under alkaline, neutral,

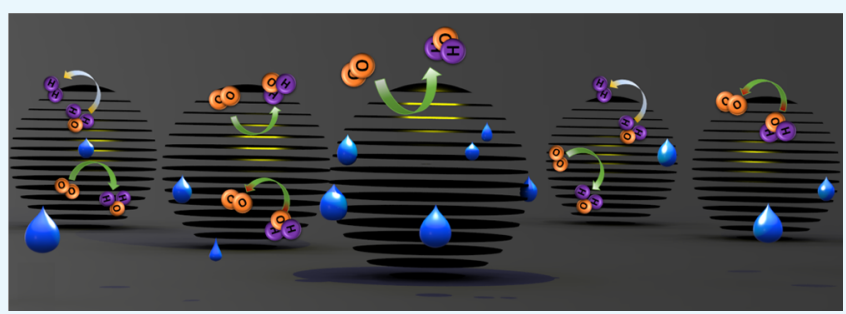
and acidic conditions. Research on $\mathrm{VSe}_{2}$ clearly demonstrates that these morphologies do not have a significant difference for ORR and OER; however, a drastic effect of morphology was observed for HER. The ORR activity of both NSs and NPs involves $\sim 2.85$ numbers of electrons with the Tafel slope of $120 \mathrm{mV} / \mathrm{dec}$ in alkaline and neutral $\mathrm{pH}$. In alkaline solution, NPs are proved to be an efficient catalyst for OER with an onset potential $1.5 \mathrm{~V}$; however, for HER, NSs have a better onset potential of - $0.25 \mathrm{~V}$. Moreover, the obtained NPs have also better catalytic activity with a $400 \mathrm{mV}$ anodic shift in the onset potential compared to NSs. These results provide a reference point for the future application of $\mathrm{VSe}_{2}$ in energy storage and conversion devices and mass production of other $2 \mathrm{D}$ materials.

\section{INTRODUCTION}

Ultrathin transition-metal dichalcogenides (TMDs) have the general formula $\mathrm{MX}_{2}$, where $\mathrm{M}$ is a transition-metal element $(\mathrm{M}=\mathrm{Ti}, \mathrm{V}, \mathrm{Ta}, \mathrm{Mo}, \mathrm{Re}, \mathrm{W})$ and $\mathrm{X}$ is a chalcogen atom $(\mathrm{X}=\mathrm{S}$, Se, Te). TMDs are one of the most promising two-dimensional (2D) layered materials ${ }^{1-3}$ as next-generation atomically thin devices and have recently become popular due to their unique properties that do not exist in their bulk counterparts. ${ }^{4,5} \mathrm{MX}_{2}$ crystal structure is based on strongly covalent (intralayer) $\mathrm{X}-$ $\mathrm{M}-\mathrm{X}$ bonds within each layer and weak van der Waals (interlayer) $\mathrm{X} \cdots \mathrm{X}$ interactions between layers. ${ }^{6}$ In recent years, broad attention has been paid to employ different types of materials and strategies for hydrogen evolution reaction $(\mathrm{HER})^{7,8}$ and oxygen evolution reaction (OER). ${ }^{9-11}$ The use of $2 \mathrm{D}$ transition-metal compounds in $\mathrm{HER}^{12-14}$ and $\mathrm{OER}^{8,15}$ applications has been the subject of many of these studies in recent years. Among all of these 2D TMDs, their semiconducting subsets, where the transition metal is typically Mo or $\mathrm{W}$ and the chalcogen atom is made of $\mathrm{S}$ or Se, have been the subject of growing attention due to their promising electrical, optoelectrical, and electrochemical characteristics. ${ }^{16-18}$

One of the most frequently employed materials for this application is molybdenum disulfide $\left(\mathrm{MoS}_{2}\right)$. It has been found that high catalytic activity of $\mathrm{MoS}_{2}$ is mainly originated from the high concentration of active sites, which are $S$ sites in this case. ${ }^{18-24}$ The hydrogen evolution reaction (HER) activity of this material is found to be linearly correlated with the concentration of these sites. ${ }^{19}$ Moreover, it is theoretically demonstrated that the activity of inert basal planes in $\mathrm{MoS}_{2}$ can be improved by adding different metal atoms, such as $\mathrm{Pt}, \mathrm{Co}$, and $\mathrm{Ni}$ dopants. ${ }^{2,25-27}$ A lot of experimental efforts have been devoted to increase the catalytic performance of the material. The introduction of high concentration of defect sites through the synthesis is one of these approaches. As already mentioned, doping with metal atoms can also enhance the HER activity of the TMD material. Phase transition from semiconductor $2 \mathrm{H}$ to metal $1 \mathrm{~T}-\mathrm{MoS}_{2}$ was also found as an efficient approach to improve the HER activity. ${ }^{28-30}$ The high electrical conductivity originated from metallic behavior in $1 \mathrm{~T}-\mathrm{MoS}_{2}$ and $1 \mathrm{~T}-\mathrm{WS}_{2}$ layers is supposed to be responsible for its high catalytic activity. However, these $1 \mathrm{~T}$ products are thermodynamically unfavorable and complicated chemical treatments are required for their synthesis. Unlike these inherently semiconducting group of TMDs that are made of group-six transition metals, group-five TMDs (where the transition metal is $\mathrm{V}, \mathrm{Nb}$, and $\mathrm{Ta}$ and the chalcogen is $\mathrm{S}, \mathrm{Se}$, and $\mathrm{Te}$ ) are purely metal and can

Received: August 22, 2017

Accepted: November 8, 2017

Published: November 27, 2017 


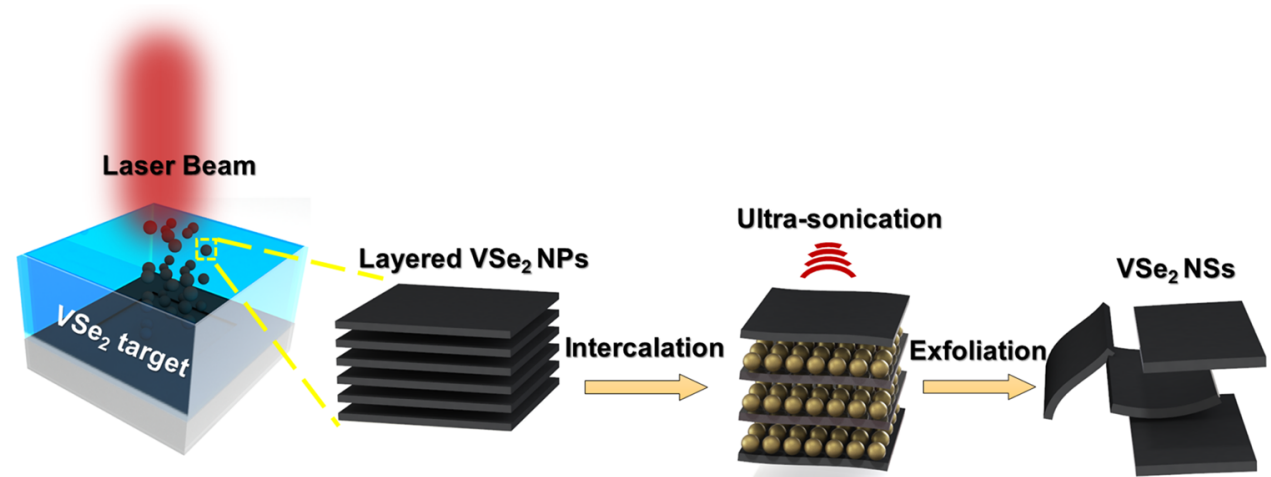

Figure 1. Proposed mechanism for exfoliated VSe $\mathrm{V}_{2} \mathrm{NS}$ synthesis: initially, stationary beam was applied to produce VSe $\mathrm{NPs}_{2}$ and then sonication is applied by adding $\mathrm{LiCO}_{3}$, which results in exfoliation of platelets of $\mathrm{VSe}_{2} \mathrm{NSs}$.

provide higher electron conductivity. ${ }^{31-33}$ Moreover, a high HER activity originating from the high activity of both basal plane and edge sites, having $\mathrm{H}$ adsorption free energy approximating to optimal $\left(\Delta G_{\mathrm{H}}=0 \mathrm{eV}\right)$, has been predicted by the density functional theory for this type of TMDs. ${ }^{34}$ In one of the first works, a superior catalytic performance was recorded for single-crystal $1 \mathrm{~T}-\mathrm{VS}_{2}$ nanosheets (NSs). The authors demonstrated that this layered TMD design can provide an extremely low overpotential of $-68 \mathrm{meV}$ at $10 \mathrm{~mA}$ / $\mathrm{cm}^{2}$, small Tafel slopes $(\sim 34 \mathrm{mV} / \mathrm{dec})$, and high stability demonstrating its potential as a candidate non-noble metal catalyst for HER ${ }^{35}$ Later, a comprehensive study ${ }^{36}$ was conducted on the catalytic performance of nine different group-five TMDs, and the findings proved that $\mathrm{VTe}_{2}$ can have even higher activity compared to $\mathrm{VS}_{2} . \mathrm{VTe}_{2}$ manifests the lowest HER overpotential at $0.5 \mathrm{~V}$ (vs reversible hydrogen electrode (RHE)) and Tafel slope of $55 \mathrm{mV} / \mathrm{dec}$. This difference was actually aroused from the morphology of the synthesized materials. As revealed in a recent study, ${ }^{39}$ lithiumexfoliated vanadium dichalcogenides $\left(\mathrm{VS}_{2}, \mathrm{VSe}_{2}, \mathrm{VTe}_{2}\right)$ were found to have dramatically different properties from their bulk counterparts. Although the HER catalytic performance of the bulk materials is $\mathrm{VTe}_{2}>\mathrm{VSe}_{2}>\mathrm{VS}_{2}$, the trend for exfoliated materials is $\mathrm{VS}_{2}>\mathrm{VSe}_{2}>\mathrm{VTe}_{2}$. These results together with previous findings suggest that as we move toward a single layer, the $\mathrm{VS}_{2}$ becomes the most active catalyst and in the bulk phase (thicker nanosheets) $\mathrm{VTe}_{2}$ is identified as the best-performing material.

Vanadium diselenide $\left(\mathrm{VSe}_{2}\right)$ is another member of this group of materials that has attracted a lot of attention as a potential 2D material for future energy storage, conversion, and sensor applications. Besides its intercalation processes ${ }^{38-40}$ and highly unusual charge density wave properties, ${ }^{41} \mathrm{VSe}_{2}$ has been predicted to have strong potential to be used as a catalyst or electrode material in electrochemical hydrogen evolution reaction, ${ }^{42}$ supercapacitors, ${ }^{43}$ photocatalytic reaction, ${ }^{44}$ and lithium-ion and sodium-ion batteries. ${ }^{45-48}$ However, there have been few studies reported on the electrochemical activities of layered $\mathrm{VSe}_{2}{ }^{39,49}$ Recently, a colloidal synthesis route to obtain single-layer $\mathrm{VSe}_{2}$ nanosheet has been developed, which offers an excellent HER activity with a low onset overpotential of $108 \mathrm{mV}$ after oxygen plasma exposure. ${ }^{49}$ A similar report revealed the potential of $\mathrm{VSe}_{2}$ for high HER performance. ${ }^{50}$ Interestingly, a very recent study proves the application of this material in photoelectrochemical application, which was unexpected before, considering the fact that the $\mathrm{VSe}_{2}$ is a metallic material with no optical band gap. ${ }^{44}$ Essentially, production of monolayer/few-layered typical 2D TMDs is mainly made possible through chemical vapor deposition $(\mathrm{CVD})^{51}$ or exfoliation from the bulk. Exfoliation can be applied either in dry phase as a mechanical cleavage ${ }^{52-54}$ (the "scotch-tape" method) from bulk crystals or in liquid phase via dispersion in a liquid, served with/without chemical reactions. ${ }^{55}$ Although mechanical exfoliation ensures high-quality and micrometer-sized flakes, it is not suitable for large-scale production because the number of layers cannot be controlled precisely. ${ }^{2}$ Chemical exfoliation of TMDs, however, is a simple and versatile method to attain nanosheets with high repeatability. ${ }^{56}$ This synthesis mainly relies on the lithiumion-assisted exfoliation and it involves two main mechanisms: (1) permeation of the $\mathrm{Li}$ ions into the planar area (intercalation) and (2) breakage of van der Waals bindings using intercalated $\mathrm{Li}^{+}$hosts (exfoliation). However, there is still a synthetic challenge to synthesize vanadium diselenide $\left(\mathrm{VSe}_{2}\right)$, which belongs to group-five layered dichalcogenides. Over the past few decades, researchers could only realize $\mathrm{VSe}_{2}$ synthesis by chemical vapor transport, ${ }^{57-59}$ chemical vapor deposition (CVD), ${ }^{51,60}$ and scotch-tape-based mechanical exfoliation ${ }^{50}$ of the bulk $\mathrm{VSe}_{2}$ until Xu et al. ${ }^{39}$ have recently synthesized it in an aqueous solution. Later, other researchers have also proposed different chemical-based synthesis methods to make $\mathrm{VSe}_{2}$ nanosheets. ${ }^{49}$ However, due to the complex chemical environment in solution, it is still challenging to prepare contamination-free samples by a solution-based method.

In the present work, we propose a top-down, large-scale compatible, surfactant-free, and widely adopted approach for the synthesis of $\mathrm{VSe}_{2}$ nanostructures (directly from bulk) by utilizing a two-stage process. In the first stage, pulsed laser ablation (PLA) technique with stationary beam scanning is applied on the bulk rock to produce $\mathrm{VSe}_{2}$ nanoparticles (NPs). Following laser ablation, ultrasonication-assisted lithium intercalation process is conducted using lithium carbonate in colloidal solution of NPs to successfully produce $\mathrm{VSe}_{2}$ nanosheets (NSs). During this process, ultrasonication assists lithium ions to easily permeate into the $\mathrm{VSe}_{2}$ matrix due to the weak van der Waals interaction between the stacked layers. The experiments are conducted to scrutinize the structural and electrochemical properties of the NP and NS structures. The electrocatalytic activities of $\mathrm{VSe}_{2} \mathrm{NPs}$ and NSs are compared to oxygen evolution reaction (OER), oxygen reduction reaction (ORR), and HER in the alkaline, neutral, and acidic media.

The practical advantage of using neutral solutions is that it is beneficial for the devices that are operated at a physiological $\mathrm{pH}$, i.e., biological and enzymatic fuel cells. ${ }^{61,62}$ For this aim, 

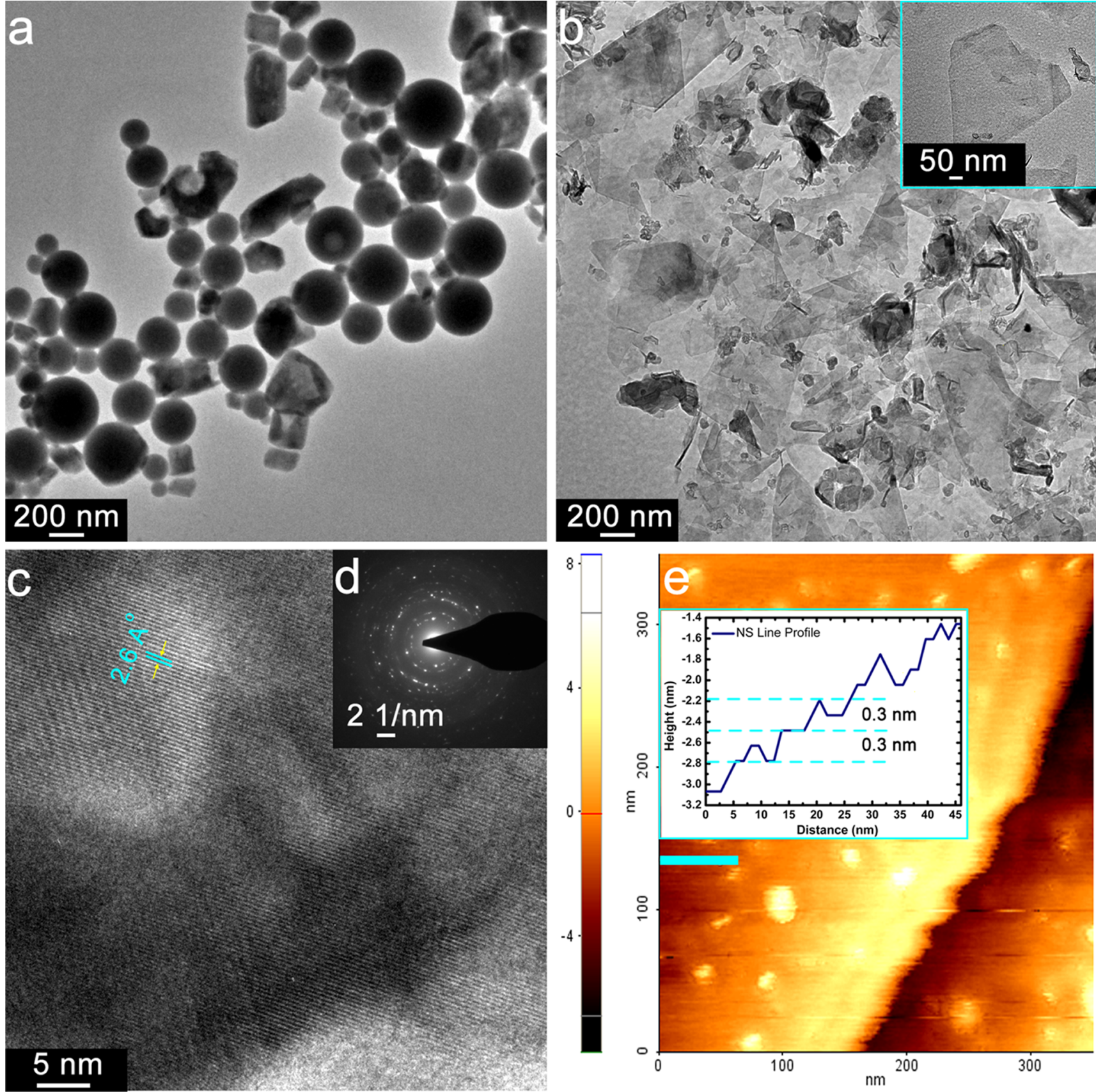

Figure 2. TEM images of (a) VSe 2 NPs obtained by nanosecond laser ablation and (b) $\mathrm{VSe}_{2} \mathrm{NSs}$ after exfoliation (inset shows a magnified image of single NS); (c) HRTEM image of $\mathrm{VSe}_{2}$ indicating $2.6 \AA$ lattice fringes, with the inset (d) showing the selected area electron diffraction (SAED) pattern; and (e) atomic force microscopy (AFM) topographic image of exfoliated NS with depth profile.

the catalytic behavior of $\mathrm{VSe}_{2}$ was evaluated under neutral conditions as well. To the best of our knowledge, this is the first report on the fabrication of $\mathrm{VSe}_{2} \mathrm{NPs}$ and NSs by employing laser ablation. Moreover, different from previous studies that are mostly focused on HER performance of the $\mathrm{VSe}_{2}$, in this study, we have conducted a comprehensive research on the OER and ORR activities of the prepared $\mathrm{VSe}_{2}$ nanostructures as well.

\section{RESULTS AND DISCUSSION}

The production of $\mathrm{VSe}_{2}$ NSs and NPs is schematically depicted in Figure 1. As shown in this figure, first, $\mathrm{VSe}_{2}$ rock target was ablated in deionized (DI) water to produce NPs with a broad size distribution. To obtain these NPs, the stationary beam was employed for 200 cycles. Afterward, $\mathrm{LiCO}_{3}$ powder was added as a lithium source to this coral-colored solution and the solution was sonicated for $90 \mathrm{~min}$ to create NSs, where the solution turns to appear as a darkish transparent solution.

For the initial step, the structural properties of the $\mathrm{VSe}_{2}$ nanostructures are investigated and shown in Figure $2 a-e$. The transmission electron microscopy (TEM) images of resultant NPs and NSs confirm that the obtained NPs by laser ablation are nearly three-dimensional (3D) spherical with high size distribution ranging from tens to hundreds of nanometers (as revealed in Figure 2a) and exfoliated layers have ultrathin 2D nature, as clearly observed in Figure $2 \mathrm{~b}$. Further investigations 

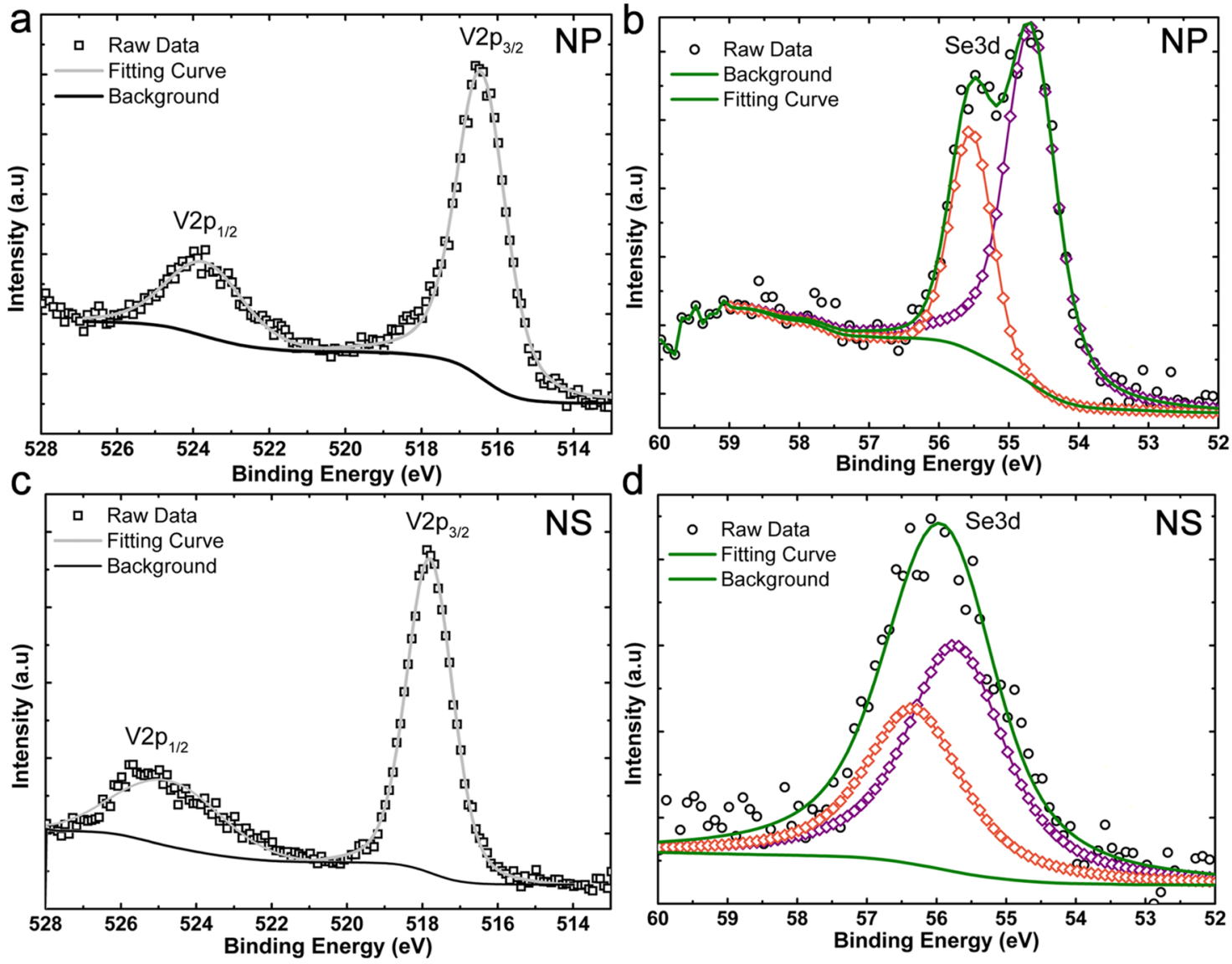

Figure 3. High-resolution XPS and their corresponding fittings: (a) V 2p and (b) Se $3 d$ for $V e_{2}$ NPs, and (c) V 2p and (d) Se $3 d$ for VSe $e_{2} N_{s}$.

using high-resolution TEM (HRTEM) images, shown in Figure 2c, prove that the fabricated NSs are single crystalline, displaying (011) lattice sets with an interplanar spacing of $2.63 \AA$ Å.

The inset located in the same figure is selected area electron diffraction (SAED) pattern taken from the NS nanostructure, and it displays a typical layered material stacked up by a few/ multiple ultrathin sheets. From these images, one can easily understand that upon dropping the $\mathrm{VSe}_{2}$ single-layer NSs on a substrate it shows an obvious tendency to self-assemble and merge into large sheets. To be more precise, the numbers of $\mathrm{VSe}_{2}$ layers are investigated by atomic force microscopy (AFM) measurements. The AFM image (Figure 2e) depicts the general observation on the exfoliated layered nature of the nanosheets and the number of layers are studied by probing the thickness; the inset shows a step profile, where the thickness of the layer increases from $\sim 0.3 \mathrm{~nm}$ (estimated thickness of the $\mathrm{VSe}_{2}$ monolayer) to $2 \mathrm{~nm}$.

For further investigations of NSs, powder X-ray diffraction (PXRD) measurements are conducted to clarify the phase and the crystalline structure of the obtained NSs. As shown in Figure S1, the structure shows a single-crystalline profile and a hexagonal phase with a dominant diffraction peak of $(011){ }^{47,63}$ On the other side, the lack of (001) main peak of $\mathrm{VSe}_{2}$ bulk can be stemmed from the laser ablation technique and we should note that the (001) and (002) peak positions in $\mathrm{VSe}_{2}$ strongly depend on the type of intercalant. ${ }^{64}$ Also, X-ray diffraction spectrum infers that the impurities are due to the presence of lithium carbonate and selenium product in the material.
To have an insight into the surface elemental composition, X-ray photoelectron spectroscopy (XPS) measurements are also carried out. An almost same peak-fitting trend is observed for both samples. Figure $3 \mathrm{a}, \mathrm{b}$ compares the deconvolution spectra of V $2 \mathrm{p}$ for NS and NP morphologies. As shown in Figure 3a, we have two distinguished peaks located at 516.7.3 $\mathrm{eV}\left(\mathrm{V} 2 \mathrm{p}_{3 / 2}\right)$ and $523.84 \mathrm{eV}\left(\mathrm{V} 2 \mathrm{p}_{1 / 2}\right)$ for NP form of $\mathrm{VSe}_{2}$. The peak at $\sim 517 \mathrm{eV}$ indicates the presence of $\mathrm{V}^{4+}$ ions, which is +4 oxidation state of vanadium. ${ }^{65}$ Figure $3 \mathrm{~b}$ shows the doublets of Se $3 \mathrm{~d}$ spectrum, Se $3 \mathrm{~d}_{5 / 2}$ and Se $3 \mathrm{~d}_{3 / 2}$ peaks located at $\sim 55.2$ and $\sim 56.1 \mathrm{eV}$ with a spin-orbit splitting of 0.86 $\mathrm{eV},{ }^{66,67}$ as it is expected; however, both $\mathrm{V} 2 \mathrm{p}$ and Se $3 \mathrm{~d}$ peaks have been slightly shifted toward higher energies in the case of NSs related to the well-known size reduction effect in metals.

As previously discussed in Introduction, TMDs provide excellent catalysts for important reactions in energy conversion and storage applications. However, to the best of our knowledge, up to now, the electrochemistry and catalytic activity of NP and NS morphologies of $\mathrm{VSe}_{2}$ have not been studied in detail. Therefore, to understand the catalytic behavior at different $\mathrm{pHs}$ toward ORR, OER, and HER, we conducted cylic voltammetry $(\mathrm{CV}) /$ linear sweep voltammetry (LSV) experiments in $0.1 \mathrm{M} \mathrm{KOH}, 0.5 \mathrm{M}$ phosphate-buffered

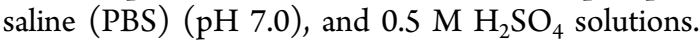

Oxygen Reduction Reaction (ORR). The ORR catalytic activities of NSs and NPs were analyzed in $\mathrm{O}_{2}$-saturated alkaline (0.1 M KOH), neutral (0.5 M PBS, $\mathrm{pH} 7.0)$, and acidic $\left(0.5 \mathrm{M} \mathrm{H}_{2} \mathrm{SO}_{4}\right)$ solutions and normalized by $\mathrm{N}_{2}$-saturated solutions shown in Figures S2-S4. Because NSs and NPs did not show any ORR catalytic activity in $\mathrm{H}_{2} \mathrm{SO}_{4}$ (data not 

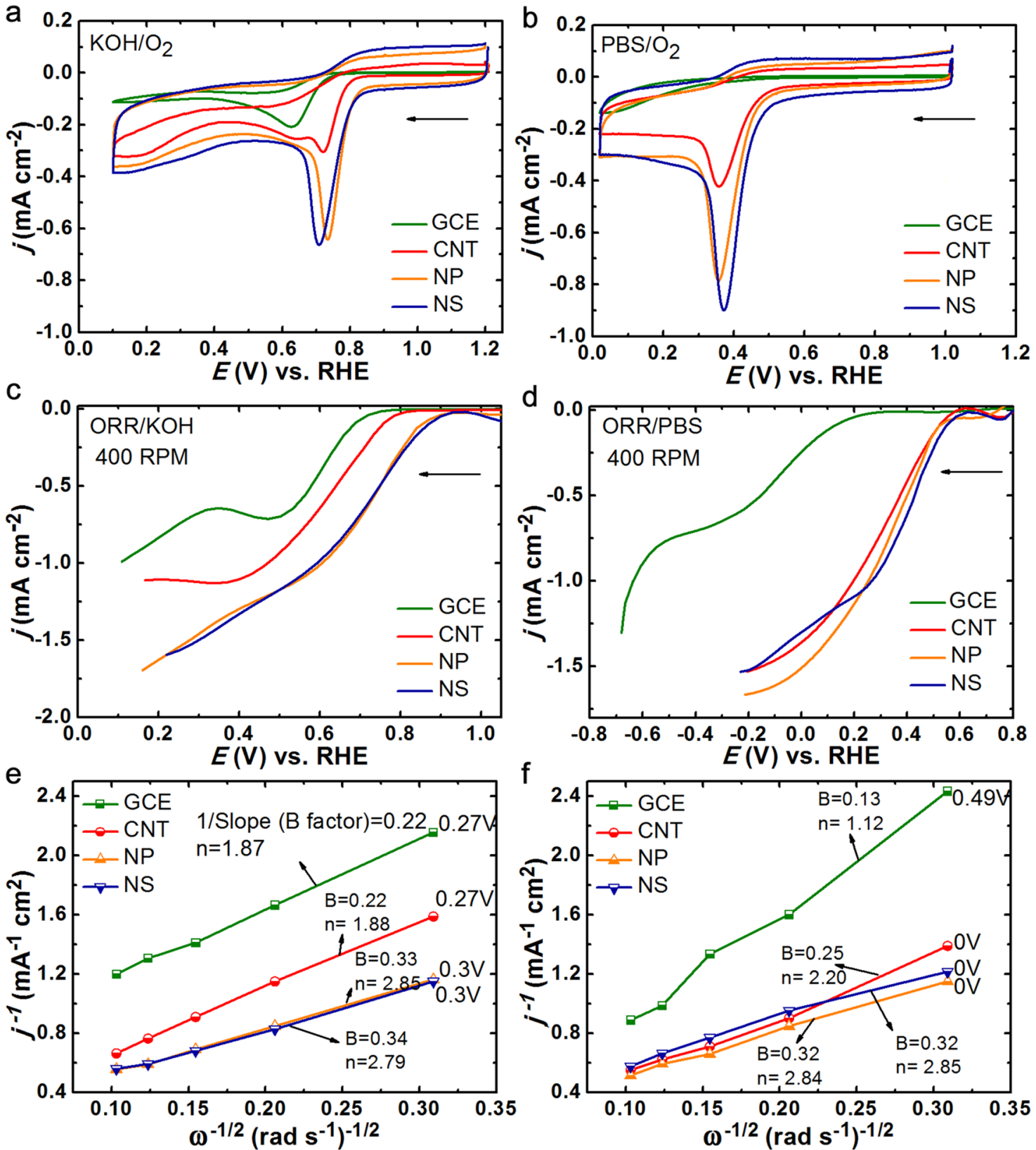

Figure 4. CVs of oxygen reduction obtained at the GCE and CNT, NP, NS catalysts in $\mathrm{O}_{2}$-saturated (a) $0.1 \mathrm{M}$ KOH solution and (b) $0.5 \mathrm{M}$ solution ( $\mathrm{pH} 7.0$ ); LSV of catalysts at $400 \mathrm{rpm}$ in $\mathrm{O}_{2}$-saturated (c) $0.1 \mathrm{M} \mathrm{KOH}$ solution and (d) $0.5 \mathrm{M} \mathrm{PBS}$ solution $(\mathrm{pH}=7.0)$ at $25^{\circ} \mathrm{C}$ at a scan rate of $10 \mathrm{mV} / \mathrm{s}$. K-L plots for catalysts in (e) $0.1 \mathrm{M} \mathrm{KOH}$ solution and (f) $0.5 \mathrm{M}$ PBS solution (pH 7.0) (data used from Figures S5 and S6).

shown), ORR in alkaline and neutral $\mathrm{pHs}$ is reported in this study. The possible reason for the noncatalytic activity of NS and $\mathrm{NP}$ in $\mathrm{H}_{2} \mathrm{SO}_{4}$ might be surface poisoning by sulfate ions. ${ }^{68-70}$ The ORR catalytic activities analyzed in $\mathrm{KOH}$ and PBS solutions using cyclic voltammograms (CVs) at $10 \mathrm{mV} / \mathrm{s}$ scan rate are plotted in Figure $4 \mathrm{a}, \mathrm{b}$, respectively. Figure $4 \mathrm{a}$ clearly shows an anodic shift in the ORR onset potentials of NS and NP in comparison to carbon nanotubes (CNTs), which are close to the $\mathrm{Pt} / \mathrm{C}$ in $\mathrm{KOH}$ solutions (see Figure S5); however, the onset potentials of NS, NP, and CNT are almost similar as the $\mathrm{pH}$ decreased to 7.0 (Figure $4 \mathrm{~b}$ ), indicating the influence of $\mathrm{pH}$ on the catalytic activity of $\mathrm{VSe}_{2}$. Consistent results were obtained in rotating disk electrode (RDE) experiments, as shown in Figure 4c,d. The RDE results at all rotations for the $\mathrm{Pt} / \mathrm{C}$, glassy carbon (GC), CNT, NP, and NS catalysts in $\mathrm{KOH}$ and PBS are plotted in Figures S5 and S6, respectively. The
Tafel slopes obtained at NS and NP cases (from Figure 4c,d) are close to $120 \mathrm{mV} / \mathrm{dec}$, which implies that the first step is a rate-limiting step for the ORR, as shown in Figure S7. Furthermore, the fitting lines in Koutecky-Levich (K-L) catalysts (Figure 4e,f) between $j^{-1}$ and $\omega^{-1}$ (where $j$ is the measured limiting current and $\omega$ is the electrode rotating rate) are plotted to estimate the number of electrons transferred per oxygen molecule $(\eta)$ involved in the ORR and related kinetics parameters (for details, see Supporting Information). In the $\mathrm{KOH}$ and PBS solutions, the number of electrons involved in the ORR at NS and NP was $\sim 2.8$, which shows that the ORR proceeds through parallel mechanism of two electrons (via $\mathrm{H}_{2} \mathrm{O}_{2}$ formation) and four electrons (direct reduction to $\mathrm{H}_{2} \mathrm{O}$ ); however, CNT undergo two-electron ORR through production of $\mathrm{H}_{2} \mathrm{O}_{2}$. In both $\mathrm{KOH}$ and PBS, exchange current density $\left(i_{0}\right)$ and kinetic current density $\left(j_{\mathrm{k}}\right)$ values were higher than those of 
Table 1. Typical ORR Results Obtained from GC, CNT, NP, and NS Catalysts Measured in 0.1 M KOH and 0.5 M PBS pH 7.0 ${ }^{a}$

$\begin{array}{lcc}\text { catalyst } & \text { solution } & \text { onset potential/m } \\ \mathrm{Pt} / \mathrm{C} & \mathrm{KOH} & 962 \\ \mathrm{GCE} & \mathrm{KOH} & 700 \\ \mathrm{CNT} & \mathrm{KOH} & 800 \\ \mathrm{NP} & \mathrm{KOH} & 932 \\ \mathrm{NS} & \mathrm{KOH} & 945 \\ \mathrm{Pt} / \mathrm{C} & \mathrm{PBS} & 645 \\ \mathrm{GCE} & \mathrm{PBS} & 316 \\ \mathrm{CNT} & \mathrm{PBS} & 545 \\ \mathrm{NP} & \mathrm{PBS} & 546 \\ \mathrm{NS} & \mathrm{PBS} & 553\end{array}$

${ }^{a}$ All of the potentials were reported versus RHE.

the CNT for NS and NP, providing catalytic activity toward ORR. These results clearly signify that NS and NP morphologies do not have a significant effect on their ORR catalytic activities. Table 1 summarizes the corresponding data for all catalysts. Essentially, the number of electrons involved in the ORR at NP and NS in the alkaline and PBS solution follows parallel $\left(k_{1}=k_{2}\right.$ and $\left.k_{3}=0\right)$ and serial $\left(k_{2}=2 \times k_{3}\right.$ and $\left.k_{1}=0\right)$ pathways shown in Scheme $1 .^{71}$ In contrast, ORR at the glassy

Scheme 1. ORR Mechanism at GCE, CNT, NP, and NS under KOH and PBS Solutions

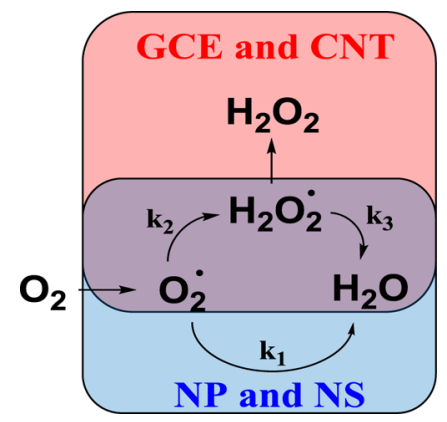

carbon electrode (GCE) and CNT follows peroxo mechanism and the serial pathway $\left(k_{2}=k_{3}\right.$ and $\left.k_{1}=0\right)$ favorably with a two-electron process. According to the Sabatier principle, ORR catalytic activity is influenced by the strength of adsorbed reactants, products, or intermediates. ${ }^{13,72}$ Thus, ORR catalysis in sulfuric acid, at the NP and NS, might be poor due to strongly adsorbed sulfate ions, which lead to weak interaction with $\mathrm{O}_{2}$ and intermediates resulting inefficient ORR catalysis.

Water Splitting: Oxygen Evolution Reaction (OER) and Hydrogen Evolution Reaction (HER). OER is a fourelectron process, and the step of water splitting is generally considered as the major kinetic obstacle of the whole water splitting due to multiple electron transfers during the formation of an $\mathrm{O}-\mathrm{O}$ bond. ${ }^{8}$ Although OER is a thermodynamically reverse process of ORR, change in the liquid to gas phase leads to a different mechanism. Some of the TMDs have already proved to be efficient catalysts for the OER of cobalt, nickel, mixed metal selenides, and so forth. ${ }^{15}$ Tahir et. al. reported that surface morphologies can change due to electrochemical treatment. ${ }^{11}$ The effect of NS and NP morphologies at different $\mathrm{pHs}$ on the OER catalytic activities were realized using $\mathrm{KOH}, \mathrm{PBS}$ ( $\mathrm{pH} 7.0$ ), and $\mathrm{H}_{2} \mathrm{SO}_{4}$ solutions. The higher onset potential obtained in OER catalysis of NS and NP in $\mathrm{H}_{2} \mathrm{SO}_{4}$ than CNT (Figure S8a) could be attributed to surface poisoning of $\mathrm{NS}$ and $\mathrm{NP}$ in $\mathrm{H}_{2} \mathrm{SO}_{4}$ solution and thus diminishing of active surface area.

Figure 5 illustrates the water splitting electrocatalytic activity of NSs and NPs in $\mathrm{KOH}$ and PBS. The catalytic activity is effective when the reaction is catalyzed at low potential (i.e., onset potentials for OER and HER shift to cathodic and anodic potentials, respectively). Figure 5a clearly shows that the onset potential for OER of NP shifts cathodically by $150 \mathrm{mV}$ in comparison to CNT, whereas NS was similar to CNT. As per convention, the potentials required for different catalysts to deliver a current density of $10 \mathrm{~mA} / \mathrm{cm}^{2}\left(E_{j=10}\right.$, overpotential value at $10 \%$ of an efficient solar water splitting device $\left.{ }^{73}\right)$ are compared and summarized in Table S2. $E_{j}$ for NP (i.e., $2.49 \mathrm{~V}$ ) was higher than the superior state-of-the-art OER catalyst $\mathrm{IrO}_{2}$ with $E_{j}=1.58 \mathrm{~V}$. Although OER is catalyzed at the NP surface, it has a high Tafel slope of $189 \mathrm{mV} / \mathrm{dec}$ (Figure S9 and Table S2), indicating slow kinetics in comparison to $\mathrm{IrO}_{2}$, but better than CNT. In the PBS solution, both the NSs and NPs are not efficient catalysts because onset potential is more anodic than that obtained for CNT (Figure 5b). On the basis of these results, it can be hypothesized that $\mathrm{OH}^{-}$ions are necessary for OER catalysis of NPs.

The HER performance of catalysts is also assessed at different $\mathrm{pHs}$ and summarized in Table S2 in addition to their catalytic activities for OER. As can be seen in the LSV curves (Figure 5c) of $\mathrm{VSe}_{2}, \mathrm{NS}$ can perform well as an outstanding HER catalyst in $1.0 \mathrm{M} \mathrm{KOH}$ electrolyte with an onset potential of about $-250 \mathrm{mV}$ (shown in Table S3), which is not as good as the commercial Pt catalyst $(243 \mathrm{mV})$, however, much better than the CNT having onset potential $-760 \mathrm{mV}$. In addition, NP also shows a similar HER catalytic activity with an onset potential of $-260 \mathrm{mV}$. Therefore, it can be concluded that $\mathrm{VSe}_{2}$ is an efficient catalyst for HER in alkaline solution. Morphology, however, does not have a significant effect on the catalytic activity in alkaline solution. The onset potential for HER shows little difference for NSs and NPs in PBS (Figure 5d). Interestingly, NPs have been proven to be an efficient HER catalyst in the $\mathrm{H}_{2} \mathrm{SO}_{4}$ solution, whereas NSs exhibit poor activity for HER with a cathodic onset potential of $400 \mathrm{mV}$ (Figure S8b). This poor catalytic behavior of NS is actually consistent with the previous results, ${ }^{37}$ where it is suggested that the degree of exfoliation can drastically affect the HER performance of the catalysts. A possible reason for the lower HER activity of NSs in $\mathrm{H}_{2} \mathrm{SO}_{4}$ is the fact that sulfate ions have stronger interaction with $2 \mathrm{D}$ NSs rather than the 3D NPs; thus, it can be removed more easily at negative potentials on the NP surface than the NS during hydrogen evolution. Thus, the morphology of catalyst plays an important role in selecting 

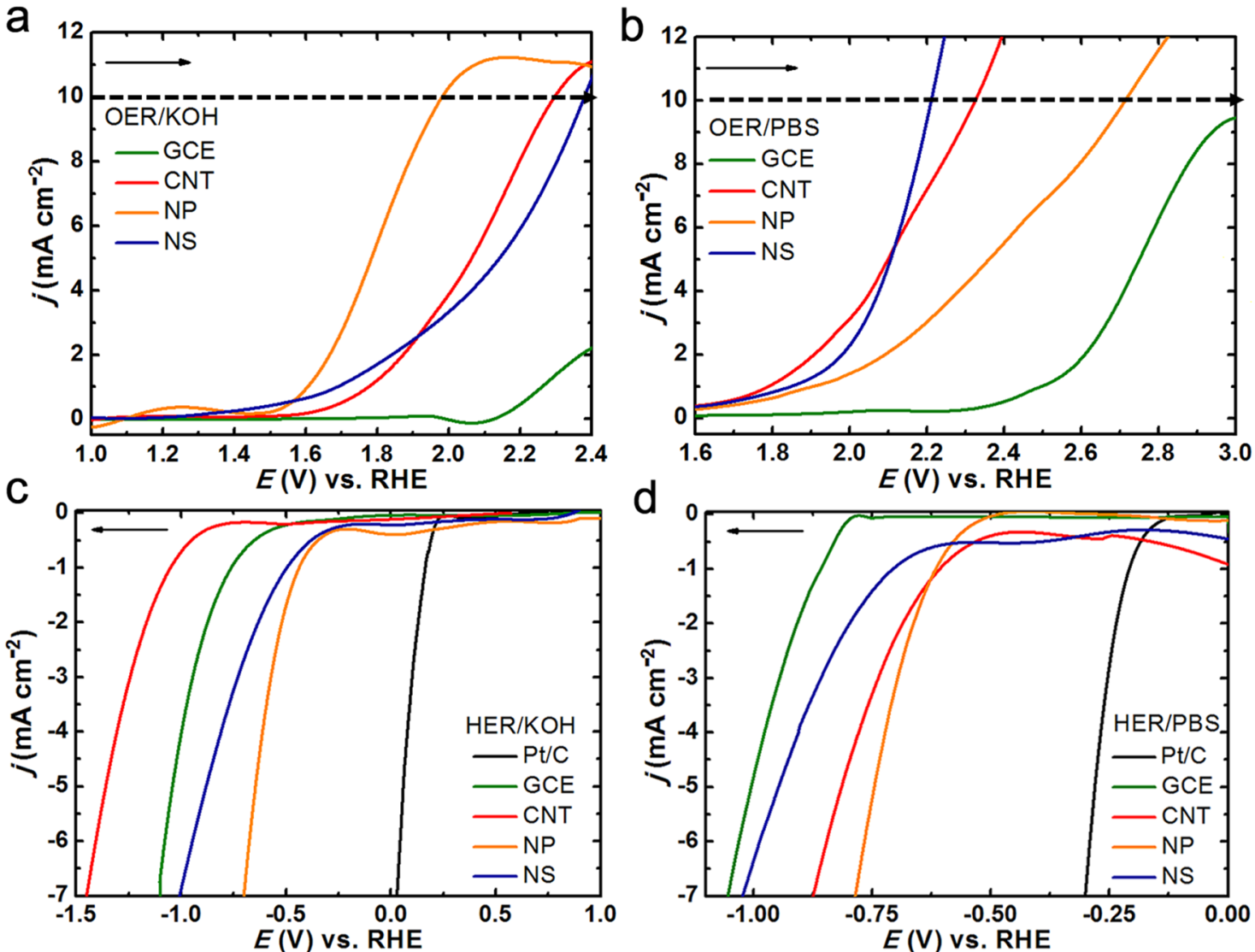

Figure 5. LSVs of OER (a, b) and HER (c, d) in $\mathrm{N}_{2}$-saturated 0.1 M KOH (a, c) and 0.5 M PBS (pH 7.0) solutions (b, d).

efficient catalyst for HER in acidic media. Revisiting the ORR and OER results, the low catalytic activity in acidic media could be attributed to the poisoning of the catalyst by $\mathrm{H}^{+}$and/or $\mathrm{SO}_{4}{ }^{2-}$ ions, which is in line with the HER catalysis results of NS; however, NP confirms its efficient catalytic activity toward $\mathrm{HER}$ in $\mathrm{H}_{2} \mathrm{SO}_{4}$. To be able to evaluate these findings, the HER process should be also revisited. Essentially, the HER reaction can proceed through the Volmer-Tafel (reactions 1 and 2) or Volmer-Heyrovsky mechanism (reactions 1 and 3). ${ }^{7,12-14,50}$ As per the nudged elastic band method, HER at TMDs follows the Volmer-Heyrovsky mechanism. ${ }^{74}$ Commonly, the first step in the HER at transition-metal selenides proceeds via hydrogen binding to selenium, which creates vacancy via desorption of hydrogen selenide. Thus, hydrogen-binding energy $\left(\Delta G_{\mathrm{H}}\right)$ and hydrogen selenide binding energy $\left(\Delta G_{\mathrm{HS}}\right)$ are important to realize catalytic activity and its stability. ${ }^{7}$

$$
\begin{aligned}
& \mathrm{M}+\mathrm{H}_{3} \mathrm{O}^{+}+\mathrm{e}^{-} \rightarrow \mathrm{M}-\mathrm{H}+\mathrm{H}_{2} \mathrm{O} \\
& 2 \mathrm{M}-\mathrm{H} \rightarrow \mathrm{H}_{2}+\mathrm{M} \\
& \mathrm{M}-\mathrm{H}+\mathrm{H}_{3} \mathrm{O}^{+}+\mathrm{e}^{-} \rightarrow \mathrm{H}_{2}+\mathrm{M}+\mathrm{H}_{2} \mathrm{O}
\end{aligned}
$$

According to the literature, $\mathrm{VSe}_{2}$ can exist as metallic TMD in either the $2 \mathrm{H}$ trigonal prismatic or $1 \mathrm{~T}$ octahedral coordination $^{60,74}$ and HER can be catalyzed at the basal planes or at the edges. Theoretically, $\Delta G_{\mathrm{HS}}$ and $\Delta G_{\mathrm{H}}$ values at $2 \mathrm{H}$ coordination have been reported as -0.57 and 0.62 for basal plane; -0.81 and 0.61 for V-edge; and -0.17 and 0.25 for Se-edge, respectively. Furthermore, $\Delta G_{\mathrm{HS}}$ and $\Delta G_{\mathrm{H}}$ values at $1 \mathrm{~T}$ coordination are -0.21 and 0.39 for basal plane and -0.17 and 0.25 for edges, respectively. ${ }^{74}$ The present study shows that
HER was catalyzed at NP and NS in the $\mathrm{KOH}$ solution; however, only $\mathrm{NP}$ in $\mathrm{H}_{2} \mathrm{SO}_{4}$ and both morphologies were inactive at neutral $\mathrm{pH}$. Thus, it clearly proves that hydrogen-ion concentration may be one of the causes to change $\Delta G_{\mathrm{HS}}$, resulting in HER catalysis being affected by $\mathrm{pH}$. The $2 \mathrm{H}$ and $1 \mathrm{~T}$ coordination influences HER catalytic activity due to the difference in their $\Delta G_{\mathrm{HS}}$ and $\Delta G_{\mathrm{H}}$ values. In the case of NP and $\mathrm{NS}$, the ratio of $2 \mathrm{H}$ and $1 \mathrm{~T}$ might be different, which can be one rational of HER catalysis at NP and inactivity of NS in $\mathrm{H}_{2} \mathrm{SO}_{4}$ solution. Thus, catalysis in $\mathrm{H}_{2} \mathrm{SO}_{4}$ can be influenced by sulfate-ion poisoning as well as the difference in the hydrogenbinding energies during the HER.

We further tested the durability of the catalysts by successive $\mathrm{CV}$ scanning at a rate of $100 \mathrm{mV} / \mathrm{s}$ for 500 cycles. As observed in Figure S10, the $\mathrm{VSe}_{2} \mathrm{NPs}$ and NSs have minor capacitive differences in all three solutions suggesting that they do not dissolve in the solution during cycling and that the overall electrochemically active area remains constant in acidic, alkaline, and neutral $\mathrm{pH}$.

\section{CONCLUSIONS}

In this work, we conducted a systematic electrochemical analysis on the $\mathrm{VSe}_{2} 2 \mathrm{D}$ nanosheets and $3 \mathrm{D}$ nanoparticles. For this aim, first, we introduced a large-scale-compatible and surfactant-free route to obtain $\mathrm{VSe}_{2}$ nanoparticles and nanosheets in high yield. In this synthesis, NPs are produced using laser ablation method. Further, we exfoliate them to obtain $\mathrm{VSe}_{2} \mathrm{NSs}$, using a Li-based intercalation assisted by sonication. In belief, the proposed synthesis method will be equally applicable to other layered TMDs, which can be used for the mass production of $2 \mathrm{D}$ nanosheets. Afterward, we performed a 
comprehensive study on the electrochemical properties of the synthesized structures and showed that the electrocatalytic activity depends on the morphology of the catalyst in alkaline, neutral, and acidic mediums. To summarize, the effect of different morphologies is noticeable only in the HER catalytic activities in $\mathrm{H}_{2} \mathrm{SO}_{4}$ solution. Both the NP and NS show ORR activity in $\mathrm{KOH}$ and PBS; however, they are inactive in the $\mathrm{H}_{2} \mathrm{SO}_{4}$ solution. NP proves to be a competent OER catalyst in $\mathrm{KOH}$, whereas a similar activity is attained by NS in the PBS solution. These results clearly demonstrate that the NS and NP shapes of the $\mathrm{VSe}_{2}$ catalyst can influence their catalytic activities depending on the $\mathrm{pH}$ of solutions. Thus, selection of proper morphology of catalyst is necessary to conclude its catalytic activity toward ORR and water splitting.

\section{EXPERIMENTAL SECTION}

Chemicals and Reagents. All chemicals were used as received without further purification. Nafion solution (Dupont DE520, 5 wt \%), lithium carbonate $\left(\mathrm{Li}_{2} \mathrm{CO}_{3}, 99.99 \%\right)$, and multiwalled carbon nanotubes (MWCNTs) were purchased from Sigma-Aldrich, and $\mathrm{VSe}_{2}$ crystal rock (>99.995\%) was supplied by HQ Graphene. Deionized water $(18 \mathrm{M} \Omega / \mathrm{cm})$ is used in all experiments.

$\mathrm{VSe}_{2}$ Nanoparticles (VSe $\mathrm{S}_{2}$ NS) Synthesis. The PLA technique was modified on the basis of our previously reported method. $^{75}$ In brief, a Nufern NuQ fiber laser (NUQA-1064NA-0030-F1) is operated at ambient temperature with 1064 $\mathrm{nm}$ wavelength, $100 \mathrm{~ns}$ pulse width duration, $30 \mathrm{kHz}$ pulse repetition rate/frequency, and $1 \mathrm{~mJ}$ pulse energy. To synthesize the $\mathrm{VSe}_{2}$ colloidal nanoparticles, $\mathrm{VSe}_{2}$ rock is used as a bulk target, which is covered by deionized water, and the water height over the target is adjusted to be $\sim 1 \mathrm{~cm}$. The laser beam is focused on the $\mathrm{VSe}_{2}$ target $(1 \mathrm{~cm}$ diameter active area), immersed in deionized water, with a spot size of about $3.8 \mathrm{~mm}$ diameter using a $200 \mathrm{~mm}$ focal length taking into account the refraction through the water. The ablation was carried out for 200 loops using the stationary beam (at a fluence amount of 30 $\mathrm{mJ} / \mathrm{cm}^{2}$ ) to produce nanoparticles with a wide size distribution $(10-100 \mathrm{~nm})$.

Lithium Intercalation of $\mathrm{VSe}_{2}$ Nanosheets (VSe $\mathrm{NSs}_{2}$. The intercalation reactions were performed by adding $10 \mathrm{mg}$ of $\mathrm{Li}_{2} \mathrm{CO}_{3}$ into $2 \mathrm{~mL}$ of $\mathrm{VSe}_{2} \mathrm{NP}$ solutions, with a molar Li excess of roughly $2: 1$. Then, the solution was dispersed by ultrasonication for $2 \mathrm{~h}$.

Ink Preparation. The slurries (ink) for $\mathrm{RDE}$ measurements were prepared by mixing $8 \mathrm{mg}$ of MWCNT, $60 \mu \mathrm{L}$ of Nafion solution ( 5 wt \%), $100 \mu \mathrm{L}$ of ethanol, $500 \mu \mathrm{L}$ of DI water, and $160 \mu \mathrm{L}$ of $\mathrm{VSe}_{2} \mathrm{NP}$ or NS solution $(\sim 16 \mathrm{mg})$ as electrocatalyst. In the absence of $\mathrm{VSe}_{2}$, ink solutions are prepared by adding the same amount of DI water $(160 \mu \mathrm{L})$ to the ink solution. Next, the sample was stored for characterization.

Materials Characterization. To characterize the structural properties of the synthesized $\mathrm{VSe}_{2} \mathrm{NPs}$ and NSs, a transmission electron microscope (Tecnai G2-F30, FEI) operated at $200 \mathrm{kV}$ was used. For TEM and high-resolution TEM (HRTEM) measurements, a few droplets of the solution were dispersed on a lacey carbon-coated copper grid. Selected area electron diffraction (SAED) patterns of samples were also collected to understand the growth direction and crystallinity of the prepared nanoparticles. X-ray photoelectron spectroscopy (XPS, Thermoscientific K-Alpha, $\mathrm{Al} \mathrm{K} \alpha$ radiation, $h v=$ $1486.6 \mathrm{eV}$ ) measurement was performed at survey mode by operating flood gun to prevent surface charging with the pass energy and step size set to 30 and $0.1 \mathrm{eV}$, respectively. The peak positions were calibrated by referencing the $\mathrm{C} 1 \mathrm{~s}$ peak position $(284.8 \mathrm{eV})$ and shifting other peaks in the spectrum accordingly. Powder X-ray diffraction (PXRD) was carried out by PANalytical X'pert Multi-Purpose Diffractometer, and the patterns were collected in the $2 \theta$ range of $10-70^{\circ}$ using Bragg-Brentano geometry $\left(\mathrm{Cu} \mathrm{K} \alpha\right.$ radiation). The $\mathrm{VSe}_{2}$ solutions were drop-cast onto the sheet of thin glass at room temperature. Finally, the height profile and layer thickness were evaluated from the tapping mode atomic force microscopy (AFM, PSIA).

Electrochemical Measurements. Electrochemical measurements were performed using a rotating disk electrode (RDE, SP-150, Bio-Logic Science Instruments) in a standard threeelectrode electrochemical cell configuration with catalyst-loaded GCE, Pt spiral wire, and $\mathrm{Ag}|\mathrm{AgCl}| \mathrm{KCl}_{(\text {sat }}$ as the working, counter, and reference electrodes, respectively. For the convenience of comparison, all potentials measured vs $\mathrm{Agl}$ $\mathrm{AgCllKCl}_{(\text {sat })}$ were reported versus RHE and all of the calculations were based on the geometric surface area, unless specified.

The GCE is renewed by polishing with $1 \mu \mathrm{m}$ diamond particle slurry and then with $0.05 \mu \mathrm{m}$ alumina powder on the polishing pad. Finally, it is washed ultrasonically with DI water and ethanol for $10 \mathrm{~min}$ each and dried at room temperature to make a homogenous film on the GC electrode surface. For performance comparison, the GCE surfaces were investigated in this study by depositing $\mathrm{Pt} / \mathrm{C}, \mathrm{CNT}, \mathrm{NP}$, and $\mathrm{NS}$ and comparing with bare GCE. As working electrode, $6 \mu \mathrm{L}$ of the catalyst ink was loaded on the GCE ( $3 \mathrm{~mm}$ diameter and $0.07068 \mathrm{~cm}^{2}$ geometric surface area) and dried at room temperature for electrochemical measurements.

The electrochemical measurements were performed in phosphate-buffered saline (PBS, 0.5 M, pH 7.02), potassium hydroxide $(0.1 \mathrm{M} \mathrm{KOH})$, and sulfuric acid $\left(0.5 \mathrm{M} \mathrm{H}_{2} \mathrm{SO}_{4}\right)$ solutions, and prior to each measurement, the electrolyte solution was saturated with either $\mathrm{N}_{2}$ or $\mathrm{O}_{2}$ gas (99.999\% purity) for $40 \mathrm{~min}$.

Oxygen Reduction Reaction (ORR). Rotating disk electrode (RDE) measurements were performed in an $\mathrm{O}_{2}$-saturated $\mathrm{KOH}$ and PBS solution to assess the ORR activity and kinetics of the $\mathrm{Pt} / \mathrm{C}, \mathrm{GCE}, \mathrm{CNT}, \mathrm{NP}$, and NS catalysts with different rotation speeds of $100,225,400,625$, and $900 \mathrm{rpm}$ at $25^{\circ} \mathrm{C}$ at a scan rate of $10 \mathrm{mV} / \mathrm{s}$.

Water Splitting: Oxygen Evolution Reaction (OER) and Hydrogen Evolution Reaction (HER). The OER and HER catalytic activities of the samples were recorded by LSVs in $\mathrm{N}_{2}$ saturated 1.0 M KOH, 0.5 M PBS, and $0.5 \mathrm{M} \mathrm{H}_{2} \mathrm{SO}_{4}$ solutions at a scan rate of $10 \mathrm{mV} / \mathrm{s}$ at room temperature.

\section{ASSOCIATED CONTENT}

\section{Supporting Information}

The Supporting Information is available free of charge on the ACS Publications website at DOI: 10.1021/acsomega.7b01226.

Detailed experimental procedures and characterization; XRD image; electrochemical performance measurement results of all electrodes (PDF)

\section{AUTHOR INFORMATION}

\section{Corresponding Author}

*E-mail: yilmaz@unam.bilkent.edu.tr. 


\section{ORCID}

T. Gamze Ulusoy Ghobadi: 0000-0002-7669-1587

\section{Present Address}

\#Dr. Okyay was at Bilkent University at the time of the study (A.K.O.).

\section{Author Contributions}

The manuscript was written through contributions of all authors. All authors have given approval to the final version of the manuscript.

\section{Notes}

The authors declare no competing financial interest.

\section{ACKNOWLEDGMENTS}

This work was partially supported by the Scientific and Technological Research Council of Turkey (TUBITAK) under Grant No. 115M375. The authors thank Asst. Prof. Serkan Kasirga for supplying material.

\section{REFERENCES}

(1) Pumera, M.; Sofer, Z.; Ambrosi, A. Layered Transition Metal Dichalcogenides for Electrochemical Energy Generation and Storage. J. Mater. Chem. A 2014, 2, 8981.

(2) Tedstone, A. A.; Lewis, D. J.; O’Brien, P. Synthesis, Properties, and Applications of Transition Metal-Doped Layered Transition Metal Dichalcogenides. Chem. Mater. 2016, 28, 1965-1974.

(3) Duan, X.; Wang, C.; Pan, A.; Yu, R.; Duan, X. Two-Dimensional Transition Metal Dichalcogenides as Atomically Thin Semiconductors: Opportunities and Challenges. Chem. Soc. Rev. 2015, 44, 8859-8876.

(4) Wasey, A. H. M. A.; Chakrabarty, S.; Das, G. P. Quantum Size Effects in Layered VX2 $(X=S, S e)$ Materials: Manifestation of Metal to Semimetal or Semiconductor Transition. J. Appl. Phys. 2015, 117, No. 064313.

(5) Wang, H.; Yuan, H.; Sae Hong, S.; Li, Y.; Cui, Y. Physical and Chemical Tuning of Two-Dimensional Transition Metal Dichalcogenides. Chem. Soc. Rev. 2015, 44, 2664-2680.

(6) Lu, T.; Dong, S.; Zhang, C.; Zhang, L.; Cui, G. Fabrication of Transition Metal Selenides and Their Applications in Energy Storage. Coord. Chem. Rev. 2017, 332, 75-99.

(7) Eftekhari, A. Electrocatalysts for Hydrogen Evolution Reaction. Int. J. Hydrogen Energy 2017, 42, 11053-11077.

(8) Wang, Y.; Kong, B.; Zhao, D.; Wang, H.; Selomulya, C. Strategies for Developing Transition Metal Phosphides as Heterogeneous Electrocatalysts for Water Splitting. Nano Today 2017, 15, 26-55.

(9) Eftekhari, A. Tuning the Electrocatalysts for Oxygen Evolution Reaction. Mater. Today Energy 2017, 5, 37-57.

(10) Anantharaj, S.; Karthick, K.; Kundu, S. Evolution of Layered Double Hydroxides (LDH) as High Performance Water Oxidation Electrocatalysts: A Review with Insights on Structure, Activity and Mechanism. Mater. Today Energy 2017, 6, 1-26.

(11) Tahir, M.; Pan, L.; Idrees, F.; Zhang, X.; Wang, L.; Zou, J. J.; Wang, Z. L. Electrocatalytic Oxygen Evolution Reaction for Energy Conversion and Storage: A Comprehensive Review. Nano Energy 2017, 37, 136-157.

(12) Hu, J.; Zhang, C.; Meng, X.; Lin, H.; Hu, C.; Long, X.; Yang, S. Hydrogen Evolution Electrocatalysis with Binary-Nonmetal Transition Metal Compounds. J. Mater. Chem. A 2017, 5, 5995-6012.

(13) Tao, H.; Gao, Y.; Talreja, N.; Guo, F.; Texter, J.; Yan, C.; Sun, Z. Two-Dimensional Nanosheets for Electrocatalysis in Energy Generation and Conversion. J. Mater. Chem. A 2017, 5, 7257-7284.

(14) Su, J.; Zhou, J.; Wang, L.; Liu, C.; Chen, Y. Synthesis and Application of Transition Metal Phosphides as Electrocatalyst for Water Splitting. Sci. Bull. 2017, 62, 633-644.

(15) Wang, M.; Lin, M.; Li, J.; Huang, L.; Zhuang, Z.; Lin, C.; Zhou, L.; Mai, L. Metal-organic Framework Derived Carbon-Confined Ni2P Nanocrystals Supported on Graphene for an Efficient Oxygen Evolution Reaction. Chem. Commun. 2017, 53, 8372-8375.
(16) Splendiani, A.; Sun, L.; Zhang, Y.; Li, T.; Kim, J.; Chim, C. Y.; Galli, G.; Wang, F. Emerging Photoluminescence in Monolayer MoS2. Nano Lett. 2010, 10, 1271-1275.

(17) Ganatra, R.; Zhang, Q. Few-Layer MoS2: A Promising Layered Semiconductor. ACS Nano 2014, 8, 4074-4099.

(18) Zhang, G.; Liu, H.; Qu, J.; Li, J. Two-Dimensional Layered MoS2: Rational Design, Properties and Electrochemical Applications. Energy Environ. Sci. 2016, 9, 1190-1209.

(19) Jaramillo, T. F.; Jorgensen, K. P.; Bonde, J.; Nielsen, J. H.; Horch, S.; Chorkendorff, I. Identification of Active Edge Sites for Electrochemical H2 Evolution from MoS2 Nanocatalysts. Science 2007, 317, 100-102.

(20) Li, G.; Zhang, D.; Qiao, Q.; Yu, Y.; Peterson, D.; Zafar, A.; Kumar, R.; Curtarolo, S.; Hunte, F.; Shannon, S.; Zhu, Y.; Yang, W.; Cao, L. All the Catalytic Active Sites of MoS2 for Hydrogen Evolution. J. Am. Chem. Soc. 2016, 138, 16632-16638.

(21) Yan, Y.; Xia, B.; Ge, X.; Liu, Z.; Wang, J. Y.; Wang, X. Ultrathin MoS2 Nanoplates with Rich Active Sites as Highly Efficient Catalyst for Hydrogen Evolution. ACS Appl. Mater. Interfaces 2013, 5, 1279412798.

(22) Wu, Z.; Fang, B.; Wang, Z.; Wang, C.; Liu, Z.; Liu, F.; Wang, W.; Alfantazi, A.; Wang, D.; Wilkinson, D. P. MoS2 Nanosheets: A Designed Structure with High Active Site Density for the Hydrogen Evolution Reaction. ACS Catal. 2013, 3, 2101-2107.

(23) Benson, J.; Li, M.; Wang, S.; Wang, P.; Papakonstantinou, P. Electrocatalytic Hydrogen Evolution Reaction on Edges of a Few Layer Molybdenum Disulfide Nanodots. ACS Appl. Mater. Interfaces 2015, 7, 14113-14122.

(24) Ambrosi, A.; Sofer, Z.; Pumera, M. Lithium Intercalation Compound Dramatically Influences the Electrochemical Properties of Exfoliated MoS2. Small 2015, 11, 605-612.

(25) Deng, J.; Li, H.; Xiao, J.; Tu, Y.; Deng, D.; Yang, H.; Tian, H.; Li, J.; Ren, P.; Bao, X. Triggering the Electrocatalytic Hydrogen Evolution Activity of the Inert Two-Dimensional MoS2 Surface via Single-Atom Metal Doping. Energy Environ. Sci. 2015, 8, 1594-1601.

(26) Escalera-López, D.; Niu, Y.; Yin, J.; Cooke, K.; Rees, N. V.; Palmer, R. E. Enhancement of the Hydrogen Evolution Reaction from Ni-MoS2 Hybrid Nanoclusters. ACS Catal. 2016, 6, 6008-6017.

(27) Wang, H.; Tsai, C.; Kong, D.; Chan, K.; Abild-Pedersen, F.; Nørskov, J. K.; Cui, Y. Transition-Metal Doped Edge Sites in Vertically Aligned MoS2 Catalysts for Enhanced Hydrogen Evolution. Nano Res. 2015, 8, 566-575.

(28) Lukowski, M. A.; Daniel, A. S.; Meng, F.; Forticaux, A.; Li, L.; Jin, S. Enhanced Hydrogen Evolution Catalysis from Chemically Exfoliated Metallic MoS2 Nanosheets. J. Am. Chem. Soc. 2013, 135, 10274-10277.

(29) Lin, Y.-C.; Dumcenco, D. O.; Huang, Y.-S.; Suenaga, K. Atomic Mechanism of the Semiconducting-to-Metallic Phase Transition in Single-Layered MoS2. Nat. Nanotechnol. 2014, 9, 391-396.

(30) Leng, K.; Chen, Z.; Zhao, X.; Tang, W.; Tian, B.; Nai, C. T.; Zhou, W.; Loh, K. P. Phase Restructuring in Transition Metal Dichalcogenides for Highly Stable Energy Storage. ACS Nano 2016, $10,9208-9215$.

(31) Feng, J.; Sun, X.; Wu, C.; Peng, L.; Lin, C.; Hu, S.; Yang, J.; et al. Metallic Few-Layered VS2 Ultrathin Nanosheets: High Two-Dimensional Conductivity for In-Plane Supercapacitors. J. Am. Chem. Soc. 2011, 133, 17832-17838.

(32) Rout, C. S.; Kim, B.; Xu, X.; Yang, J.; Jeong, H. Y.; Odkhuu, D.; Park, N.; Cho, J.; Shin, H. S. Synthesis and Characterization of Patronite Form of Vanadium Sulfide on Graphitic Layer. J. Am. Chem. Soc. 2013, 135, 8720-8725.

(33) Jing, Y.; Zhou, Z.; Cabrera, C. R.; Chen, Z. Metallic VS2 Monolayer: A Promising 2D Anode Material for Lithium Ion Batteries. J. Phys. Chem. C 2013, 117, 25409-25413.

(34) Li, H.; Tsai, C.; Koh, A. L.; Cai, L.; Contryman, A. W.; Fragapane, A. H.; Zhao, J.; Han, H. S.; Manoharan, H. C.; AbildPedersen, F.; Nørskov, J. K.; Zheng, X. Activating and Optimizing MoS2 Basal Planes for Hydrogen Evolution through the Formation of Strained Sulphur Vacancies. Nat. Mater. 2016, 15, 364. 
(35) Yuan, J.; Wu, J.; Hardy, W. J.; Loya, P.; Lou, M.; Yang, Y.; Najmaei, S.; Jiang, M.; Qin, F.; Keyshar, K.; Ji, H.; Gao, W.; Bao, J.; Kono, J.; Natelson, D.; Ajayan, P. M.; Lou, J. Facile Synthesis of Single Crystal Vanadium Disulfi de Nanosheets by Chemical Vapor Deposition for Efficient Hydrogen Evolution Reaction. Adv. Mater. 2015, 27, 5605-5609.

(36) Chia, X.; Ambrosi, A.; Lazar, P.; Sofer, Z.; Pumera, M. Electrocatalysis of Layered Group 5 Metallic Transition Metal Dichalcogenides (MX2, M $=\mathrm{V}, \mathrm{Nb}$, and Ta; $\mathrm{X}=\mathrm{S}$, Se, and Te). J. Mater. Chem. A 2016, 4, 14241-14253.

(37) Wang, Y.; Sofer, Z.; Luxa, J.; Pumera, M. Lithium Exfoliated Vanadium Dichalcogenides (VS2, VSe2, VTe2) Exhibit Dramatically Different Properties from Their Bulk Counterparts. Adv. Mater. Interfaces 2016, 3, No. 1600433.

(38) Reshak, A. H.; Auluck, S. Theoretical Investigations of the Electronic and Optical Properties of Pure and Alkali Metal Intercalated 1T-VSe2. Phys. B 2004, 349, 310-315.

(39) Xu, K.; Chen, P.; Li, X.; Wu, C.; Guo, Y.; Zhao, J.; Wu, X.; Xie, Y. Ultrathin Nanosheets of Vanadium Diselenide: A Metallic TwoDimensional Material with Ferromagnetic Charge-Density-Wave Behavior. Angew. Chem., Int. Ed. 2013, 52, 10477-10481.

(40) Brauer, H. E.; Ekvall, I.; Olin, H.; Starnberg, H. I.; Wahlström, E.; Hughes, H. P.; Strocov, V. N. Na Intercalation of $\mathrm{VSe}_{2}$ Studied by Photoemission and Scanning Tunneling Microscopy. Phys. Rev. B 1997, 55, 10022-10026.

(41) Yadav, C. S.; Rastogi, A. K. Electronic Transport and Specific Heat of 1T-VSe2. Solid State Commun. 2010, 150, 648-651.

(42) Chhowalla, M.; Shin, H. S.; Eda, G.; Li, L.-J.; Loh, K. P.; Zhang, $\mathrm{H}$. The Chemistry of Two-Dimensional Layered Transition Metal Dichalcogenide Nanosheets. Nat. Chem. 2013, 5, 263-275.

(43) Marri, S. R.; Ratha, S.; Rout, C. S.; Behera, J. N. 3D Cuboidal Vanadium Diselenide Embedded Reduced Graphene Oxide Hybrid Structures with Enhanced Supercapacitor Properties. Chem. Commun. 2017, 53, 228-231.

(44) He, S.; Lin, H.; Qin, L.; Mao, Z.; He, H.; Li, Y.; Li, Q. Synthesis, Stability and Intrinsic Photocatalytic Properties of Vanadium Diselenide. J. Mater. Chem. A 2017, 5, 2163-2171.

(45) Yang, X.; Zhang, Z. Carbon-Coated Vanadium Selenide as Anode for Lithium-Ion Batteries and Sodium-Ion Batteries with Enhanced Electrochemical Performance. Mater. Lett. 2017, 189, 152155.

(46) Ōnuki, Y.; Inada, R.; Tanuma, S. I.; Yamanaka, S.; Kamimura, H. Electrochemical Characteristics of TiS2, ZrSe2 and VSe2 in Secondary Lithium Battery. Jpn. J. Appl. Phys. 1981, 20, 1583-1588.

(47) Wang, Y.; Qian, B.; Li, H.; Liu, L.; Chen, L.; Jiang, H. VSe2/ graphene Nanocomposites as Anode Materials for Lithium-Ion Batteries. Mater. Lett. 2015, 141, 35-38.

(48) Murphy, D. W.; Carides, J. N. Low Voltage Behavior of Lithium/Metal Dichalcogenide Topochemical Cells. J. Electrochem. Soc. 1979, 126, 349-351.

(49) Zhao, W.; Dong, B. H.; Guo, Z. L.; Su, G.; Gao, R. J.; Wang, W.; Cao, L. X. Colloidal Synthesis of VSe2 Single-Layer Nanosheets as Novel Electrocatalysts for the Hydrogen Evolution Reaction. Chem. Commun. 2016, 52, 9228-9231.

(50) Yan, M.; Pan, X.; Wang, P.; Chen, F.; He, L.; Jiang, G.; Wang, J.; Liu, J. Z.; Xu, X.; Liao, X.; Yang, J.; Mai, L. Field-Effect Tuned Adsorption Dynamics of VSe2 Nanosheets for Enhanced Hydrogen Evolution Reaction. Nano Lett. 2017, 17, 4109-4115.

(51) Boscher, N. D.; Blackman, C. S.; Carmalt, C. J.; Parkin, I. P.; Prieto, A. G. Atmospheric Pressure Chemical Vapour Deposition of Vanadium Diselenide Thin Films. Appl. Surf. Sci. 2007, 253, 60416046.

(52) Li, H.; Wu, J.; Yin, Z.; Zhang, H. Preparation and Applications of Mechanically Exfoliated Single-Layer and Multilayer MoS2 and WSe2 Nanosheets. Acc. Chem. Res. 2014, 47, 1067-107.

(53) Mak, K. F.; Lee, C.; Hone, J.; Shan, J.; Heinz, T. F. Atomically Thin MoS2:A New Direct-Gap Semiconductor. Phys. Rev. Lett. 2010, 105, No. 136805.
(54) Hollensteiner, S.; Spiecker, E.; Dieker, C.; Jäger, W.; Adelung, R.; Kipp, L.; Skibowski, M. Self-Assembled Nanowire Formation during $\mathrm{Cu}$ Deposition on Atomically Flat Vse2 Surfaces Studied by Microscopic Methods. Mater. Sci. Eng., C 2003, 23, 171-179.

(55) Bernal, M. M.; Álvarez, L.; Giovanelli, E.; Arnáiz, A.; RuizGonzález, L.; Casado, S.; Granados, D.; Pizarro, A. M.; CastellanosGomez, A.; Pérez, E. M. Luminescent Transition Metal Dichalcogenide Nanosheets through One-Step Liquid Phase Exfoliation. 2D Mater. 2016, 3, No. 035014.

(56) Zhou, K.-G.; Mao, N.; Wang, H.; Peng, Y.; Zhang, H. A MixedSolvent Strategy for Efficient Exfoliation of Inorganic Graphene Analogues. Angew. Chem., Int. Ed. 2011, 50, 10839-10842.

(57) Nikonov, K. S.; Brekhovskikh, M. N.; Egorysheva, A. V.; Menshchikova, T. K.; Fedorov, V. A. Chemical Vapor Transport Growth of vanadium(IV) Selenide and vanadium(IV) Telluride Single Crystals. Inorg. Mater. 2017, 53, 1126-1130.

(58) Wiegers, G. A. The Characterisation of VSe2: A Study of the Thermal Expansion. J. Phys. C: Solid State Phys. 1981, 14, 4225-4235.

(59) Spiecker, E.; Schmid, A. K.; Minor, A. M.; Dahmen, U.; Hollensteiner, S.; Jäger, W. Self-Assembled Nanofold Network Formation on Layered Crystal Surfaces during Metal Intercalation. Phys. Rev. Lett. 2006, 96, No. 086401.

(60) Zhang, Z.; Niu, J.; Yang, P.; Gong, Y.; Ji, Q.; Shi, J.; Fang, Q.; Jiang, S.; Li, H.; Zhou, X.; Gu, L.; Wu, X.; Zhang, Y. Van Der Waals Epitaxial Growth of 2D Metallic Vanadium Diselenide Single Crystals and Their Extra-High Electrical Conductivity. Adv. Mater. 2017, 29, No. 1702359

(61) Shimizu, K.; Sepunaru, L.; Compton, R. G. Innovative Catalyst Design for Oxygen Reduction Reaction for Fuel Cells. Chem. Sci. 2016, 7, 3364-3369.

(62) Falk, M.; Blum, Z.; Shleev, S. Direct Electron Transfer Based Enzymatic Fuel Cells. Electrochim. Acta 2012, 82, 191-202.

(63) Gopalakrishnan, J. Insertion/extraction of Lithium and Sodium in Transition Metal Oxides and Chalcogenides. Bull. Mater. Sci. 1985, 7, 201-214.

(64) Acerce, M.; Voiry, D.; Chhowalla, M. Metallic 1T Phase MoS2 Nanosheets as Supercapacitor Electrode Materials. Nat. Nanotechnol. 2015, 10, 313-318.

(65) Karamat, S.; Rawat, R. S.; Lee, P.; Tan, T. L.; Ke, C.; Chen, R.; Sun, H. D. Ferromagnetic Signature in Vanadium Doped $\mathrm{ZnO}$ Thin Films Grown by Pulsed Laser Deposition. J. Mater. Res. 2016, 31, 3223-3229.

(66) Cui, Y.; Abouimrane, A.; Lu, J.; Bolin, T.; Ren, Y.; Weng, W.; Sun, C.; Maroni, V. A.; Heald, S. M.; Amine, K. (De)lithiation Mechanism of $\mathrm{Li} / \mathrm{SeSx}(\mathrm{X}=0-7)$ Batteries Determined by in Situ Synchrotron X-Ray Diffraction and X-Ray Absorption Spectroscopy. J. Am. Chem. Soc. 2013, 135, 8047-8056.

(67) Zhang, J.; Fan, L.; Zhu, Y.; Xu, Y.; Liang, J.; Wei, D.; Qian, Y. Selenium/interconnected Porous Hollow Carbon Bubbles Composites as the Cathodes of Li-Se Batteries with High Performance. Nanoscale 2014, 6, 12952-12957.

(68) Saleh, M. M.; Awad, M. I.; Kitamura, F.; Ohsaka, T. Sulphur Dioxide Poisoning and Recovery of Platinum Nanoparticles: Effect of Particle Size. Int. J. Electrochem. Sci. 2012, 7, 12004-12020.

(69) Hamzehlouyan, T.; Sampara, C.; Li, J.; Kumar, A.; et al. Sulfur Poisoning of a Pt/Al2O3 Oxidation Catalyst: Understanding of $\mathrm{SO} 2$, SO3 and H2SO4 Impacts. Top. Catal. 2016, 59, 1028-1032.

(70) Barbarossa, V.; Brutti, S.; Brunetti, B.; Diamanti, M.; Ricci, G. Study of I-/I2 Poisoning of Fe2O3-Based Catalysts for the H2SO4 Decomposition in the Sulfur-Iodine Cycle for Hydrogen. Ind. Eng. Chem. Res. 2009, 48, 625-631.

(71) Mishra, R.; Patil, B.; Karadaş, F.; Yılmaz, E. Bioinspired Copper Coordination Polymer Catalysts for Oxygen Reduction Reaction. ChemistrySelect 2017, 2, 8296-8300.

(72) Rothenberg, B. G. Catalysis: Concepts and Green Applications, 2nd ed.; Wiley-VCH Press: Chichester, UK, 2008; p 290.

(73) McCrory, C. C. L.; Jung, S.; et al. Benchmarking Heterogeneous Electrocatalysts for the Oxygen Evolution Reaction. J. Am. Chem. Soc. 2013, 135, 16977-16987. 
(74) Tsai, C.; Chan, K.; Nørskov, J. K.; Abild-Pedersen, F. Theoretical Insights into the Hydrogen Evolution Activity of Layered Transition Metal Dichalcogenides. Surf. Sci. 2015, 640, 133-140.

(75) Ulusoy Ghobadi, T. G.; Ghobadi, A.; Okyay, T.; Topalli, K.; Okyay, A. K. Controlling Luminescent Silicon Nanoparticle Emission Produced by Nanosecond Pulsed Laser Ablation: Role of Interface Defect States and Crystallinity Phase. RSC Adv. 2016, 6, 112520112526. 